\title{
The contribution of food groups to energy and nutrient intakes in children aged 1-3 years attending a childcare centre in Dublin
}

\author{
J. L. O’Neill ${ }^{1}$, M. C. Walsh ${ }^{1}$, J. O’Dea ${ }^{1}$, E. R. Gibney ${ }^{2}$, A. Regan ${ }^{1}$ and E. Shannon ${ }^{1}$ \\ ${ }^{1}$ Danone Baby Nutrition, Block 1 Deansgrange Business Park, Deansgrange, Co. Dublin, Republic of Ireland and ${ }^{2}$ UCD \\ Institute of Food and Health, University College Dublin, Belfield, Dublin 4, Republic of Ireland
}

Diet in the early years is an important modifiable factor to ensure that children receive the right balance of foods and nutrients to support healthy growth and development ${ }^{(1)}$.

The main aim of this study was to investigate the contribution of food groups to the energy and macronutrient intakes of 40 healthy preschool children aged 1-3 years and then examine energy and nutrient intakes across tertiles of whole cows' milk intake. Parents/guardians provided written consent for their child to take part in the study. Food and beverage intake was recorded by parents/guardians/staff as appropriate, using a 3-day food diary. Dietary intake data was analysed using Dietplan 6 (Forestfield Software Ltd, UK) ${ }^{(2)}$.

\begin{tabular}{ll}
\hline$(n=40)$ & Percentage contribution of the top 3 food groups to energy and macronutrient intake \\
Energy & Whole cows' milk $(18 \%)$, Meat, meat dishes \& products $(13 \%)$, Breakfast cereals $(10 \%)$ \\
Protein & Meat, meat dishes \& products $(28 \%)$, Whole cows milk $(22 \%)$, Breakfast cereals $(7 \%)$ \\
Total Fat & Whole cows' milk $(28 \%)$, Meat, meat dishes \& products $(16 \%)$, Cheese $(7 \%)$ \\
Saturated Fat & Whole cows' milk $(37 \%)$, Meat, meat dishes \& products $(14 \%)$, Cheese $(9 \%)$ \\
Carbohydrate & Fruit \& fruit juices $(19 \%)$, Rice \& pasta $(15 \%)$, Whole cows' milk $(13 \%)$ \\
\hline
\end{tabular}

This study found that whole cows' milk is one of the main contributors to energy and macronutrient intakes, in particular saturated fat intake $(37 \%)$. Furthermore, increasing consumption of whole cows' milk can negatively contribute towards displacing the intake of key nutrients in the toddler diet. Further analysis of a small sub-group who also consumed a toddler specific fortified milk, showed a trend towards more favourable intakes of key nutrients.

\begin{tabular}{|c|c|c|c|c|c|c|c|c|}
\hline & \multicolumn{6}{|c|}{ Intake of whole cows' milk (co nsumers, $n=38$ ) } & \multicolumn{2}{|c|}{ Toddler milk (consumers, $n=10$ ) } \\
\hline & \multicolumn{2}{|c|}{ Low } & \multicolumn{2}{|c|}{ Medium } & \multicolumn{2}{|c|}{ High } & \multirow[b]{2}{*}{ Mean } & \multirow[b]{2}{*}{ SD } \\
\hline & Mean & SD & Mean & SD & Mean & SD & & \\
\hline Total intake: 3 days $(\mathrm{ml})$ & 418.8 & 198.6 & 970.2 & 125.6 & 1577.8 & 464 & 607.0 & 292.4 \\
\hline $\begin{array}{l}\text { Energy and Macronutrients } \\
\text { Energy (kcal) }\end{array}$ & 1087.1 & 153.5 & 1237.1 & 225.3 & 1231 & 308.7 & 1239.2 & 170.4 \\
\hline Protein $(\mathrm{g})$ & 43.4 & 5.8 & 48.1 & 7.2 & 49.3 & 9.7 & 49.4 & 6.3 \\
\hline Fat (\% Energy) & 30.8 & 3.8 & 35.3 & 6.1 & 35.8 & 4.6 & 32.4 & 3.9 \\
\hline Saturated Fat ( $\%$ Energy) & 14.3 & 2.2 & 15.9 & 2.5 & 17.9 & 1.8 & 14.0 & 2.6 \\
\hline Carb ohydrate ( $\%$ Energy) & 50.9 & 3.4 & 45 & 7.2 & 45.5 & 6.1 & 49.0 & 3.1 \\
\hline Micronutrients $^{\wedge}$ & & & & & & & & \\
\hline Sodium (mg) & 1144.3 & 249.1 & 1254 & 256.9 & 1338.4 & 468.3 & 1205.9 & 287.4 \\
\hline Calcium (mg) & 767.9 & 218.7 & 846.2 & 240.3 & 944.5 & 232.4 & 941.7 & 270.8 \\
\hline Iron (mg) & 8.4 & 2.3 & 8.6 & 3.6 & 7.8 & 3.5 & 10.9 & 2.7 \\
\hline Vitamin D (ug) & 4.5 & 3.9 & 2.1 & 1.7 & 1.8 & 2 & 6.5 & 4.2 \\
\hline Vitamin C (mg) & 72.9 & 33.5 & 59.6 & 24.8 & 57.7 & 30.6 & 105.4 & 33.8 \\
\hline
\end{tabular}

$\wedge$ All sources including di et ary supplements.

Identifying the underlying factors associated with energy and nutrient intake patterns in the diets of toddlers aged 1-3 years, is vital for the development of effective food-based treatment and prevention strategies.

1. Department of Health and Children. Food and Nutritional Guidelines for Pre-school Services. Dublin: Health Promotion Unit, 2004. Available from: www.dohc.ie.

2. Food Standards Agency. McCance and Widdowson's The Composition of Foods, $6^{\text {th }}$ Edition. Cambridge: Royal Society of Chemistry, 2002. 